\title{
Giant Subclavian Artery Aneurysm
}

\author{
Sarah Counts, DO ${ }^{1}$ Ahmad Zeeshan, MBBS$^{1} \quad$ John Elefteriades, MD $^{1}$ \\ ${ }^{1}$ Department of Surgery, Yale University School of Medicine, New \\ Haven, Connecticut \\ Address for correspondence Sarah Counts, DO, Department of Surgery, \\ 20 York Street, New Haven, CT, 2037857890, \\ (e-mail: Sarah.Counts@Yale.edu).
}

Int J Angiol 2016;25:128-130.

\author{
Abstract \\ Keywords \\ - subclavian artery \\ aneurysm \\ - subclavian \\ - aneurysm \\ - bicuspid aortic valve
}

We report the case of a 37-year-old construction executive presenting with chest pain, shortness of breath, and dizziness on exertion secondary to a giant left subclavian artery aneurysm and aortic valvular disease.
A 37-year-old male patient presented with dyspnea, chest pain, and episodic dizziness to the point of near syncope on exertion. The patient reported that he was known to have a murmur since he was 2 years old. On clinical examination, systolic and diastolic murmurs could be auscultated. There was no family history of significant cardiac disease, aneurysm, or premature sudden death. Physical examination revealed a $4 / 6$ systolic ejection murmur that radiated to the right greater than left carotid and a 2/6 diastolic murmur heard at the upper sternal borders accentuated with forced exhalation. The remainder of his clinical examination and his laboratory tests were unremarkable.

The workup included a chest X-ray, echocardiogram, computed tomographic angiography, and aneurysmal survey which did not show any evidence of additional aneurysmal disease elsewhere in the body. The echocardiogram revealed severe aortic stenosis with a gradient of $54 \mathrm{~mm} \mathrm{Hg}$ and the valve velocity of $3.7 \mathrm{~m} / \mathrm{sec}$, severe aortic insufficiency, a bicuspid aortic valve, severe left ventricular hypertrophy, left atrial dilatation, and an enlarged ascending aorta. CT imaging showed a bicuspid aortic valve with thickened, fused right and noncoronary cusps in addition to a nodular density immediately distal to the cusps. The ascending aorta was noted to be enlarged to $4.1 \mathrm{~cm}$ in diameter. The aortic dilatation continued into the arch. The left subclavian artery was also dilated at the takeoff from the aorta and measured $4.2 \mathrm{~cm}$ at its origin (-Figs. 1 and 2). The right brachiocephalic artery was abnormal and measured $2.3 \mathrm{~cm}$ in its proximal diameter (-Figs. 3 and 4). The left vertebral artery took off from the dilated left subclavian artery and was smaller in caliber compared with the right vertebral artery. Coronary imaging disclosed an absent left circumflex with a diagonal vessel supplying the lateral wall, with no significant stenosis or calcifications was noted.

A two-stage procedure was undertaken. The aortic valve and ascending aorta were addressed first. Intraoperative transesophageal echocardiography confirmed the findings noted in preoperative echocardiography. The ascending aorta was noted to be very small, measuring $20 \mathrm{~mm}$ at the level of the sinotubular junction, and exceptionally thin walled. The aorta was opened longitudinally and transected above the coronary arteries allowing visualization of the markedly deformed bicuspid aortic valve and annular distortion. There was a $2 \mathrm{~cm}$ nodular atheroma originating in the aortic valve and attached to the aortic wall; the valve was nearly unicuspid on direct inspection. The abnormal leaflets were removed, the annulus debrided, and a St. Jude mechanical aortic valve (St. Jude Medical Inc., St. Paul, MN) placed with interrupted pledgeted sutures. The ascending aorta was replaced with a $20 \mathrm{~mm}$ Hemashield graft (Atrium Medical Corporation, Hudson, $\mathrm{NH}$ ), with sparing of the aortic root. Total bypass time was 109 minutes. The patient did well postoperatively.

Four weeks later, the patient was taken back to the operating room to address the left subclavian artery aneurysm. Intraoperative transesophageal echocardiography revealed a competent mechanical aortic valve with no abnormalities. A left thoracotomy was performed and the published online August 19, 2014
Copyright @ 2016 by Thieme Medical Publishers, Inc., 333 Seventh Avenue, New York, NY 10001, USA. Tel: +1(212) 584-4662.
DOI http://dx.doi.org/ 10.1055/s-0034-1372245. ISSN 1061-1711. 


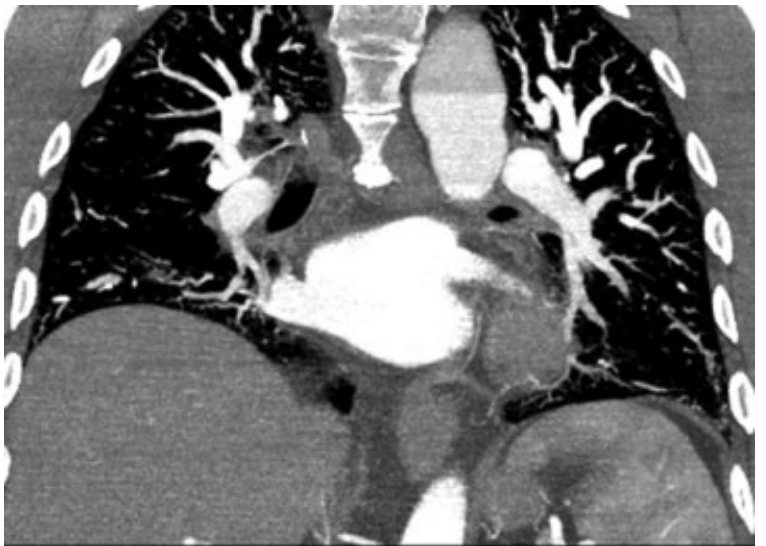

Fig. 1 Preoperative computed tomographic angiography of chest. Note large subclavian aneurysm rising vertically in the posterior chest.

entire massive subclavian artery aneurysm and aortic arch were mobilized. The subclavian artery was essentially like another aorta going up to the apex of the chest (in fact, it was larger than the descending aorta). A large aneurysm clamp was placed across the greater curvature of the aortic arch at the origin of subclavian artery that measured $5 \mathrm{~cm}$. The left subclavian artery was transected next to the clamp and the proximal stump was oversewn in a running fashion in two layers along the length of the large aneurysm clamp. The proximal anastomosis for a left subclavian artery graft was constructed at the junction of the proximal and middle thirds of the descending aorta with a $10 \mathrm{~mm}$ Hemashield graft. The distal subclavian artery was transected as high as possible and was noted to be very thin walled and approximately 12 to $14 \mathrm{~mm}$ in diameter. A beveled distal anastomosis was performed with the Hemashield graft (-Fig. 5). At the completion of the operation, a pericardial effusion from the first operation was drained with a pericardial window. The postoperative course was uneventful and he was discharged on 5 th postoperative day.

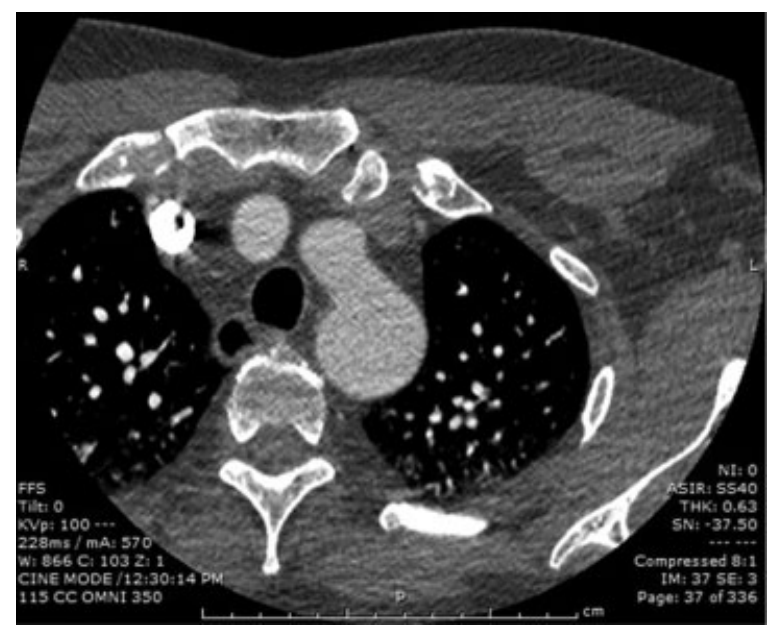

Fig. 2 Preoperative computed tomographic angiography of chest. Note subclavian aneurysm that dwarfs the native aorta.

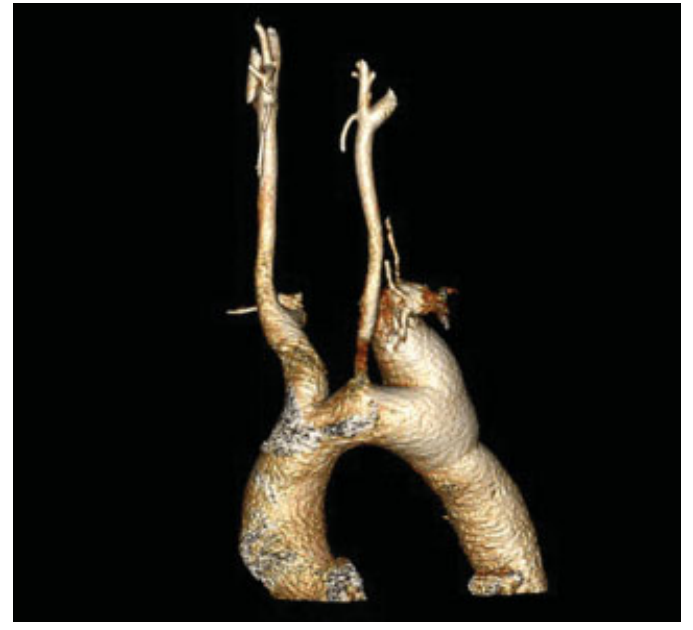

Fig. 3 Preoperative computed tomographic angiography of chest threedimensional image, anterior view. Note large subclavian aneurysm.

\section{Discussion}

Subclavian artery aneurysms are rare peripheral aneurysms accounting for 0.13 to $0.5 \%$ of all aneurysms. ${ }^{1}$ The association of left subclavian artery aneurysm with a congenital bicuspid aortic valve and aneurysm of the aorta is extremely rare. ${ }^{2}$ In an extensive review of the literature between 1926 and 1995, Dougherty et al found 70 published reports of subclavian artery aneurysms in 64 patients. Intrathoracic location was uncommonly encountered, seen in 35 cases. The average age at diagnosis was 49 years, with right-sided location twice as common as left-sided. Males were noted to be two times as likely as females to have this rare vascular abnormality. ${ }^{3}$

The most common causes of subclavian artery aneurysm are atherosclerosis, followed by infection (mycotic, syphilitic, and tuberculosis), thoracic outlet syndrome, trauma, Marfan syndrome, Ehlers-Danlos syndrome, neurofibromatosis, and congenital anomalies (Turner syndrome). ${ }^{4}$ These aneurysms range from incidentally discovered asymptomatic disease

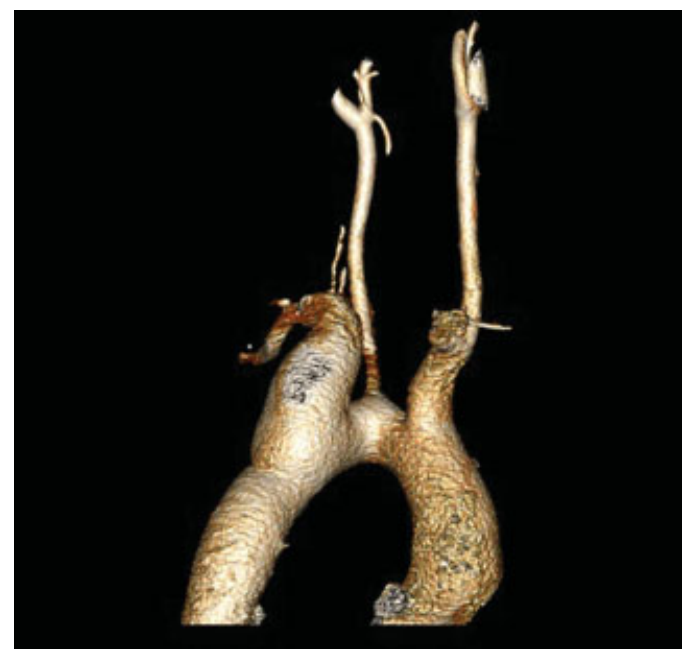

Fig. 4 Preoperative computed tomographic angiography of chest three-dimensional image, posterior view. Note large subclavian aneurysm. 


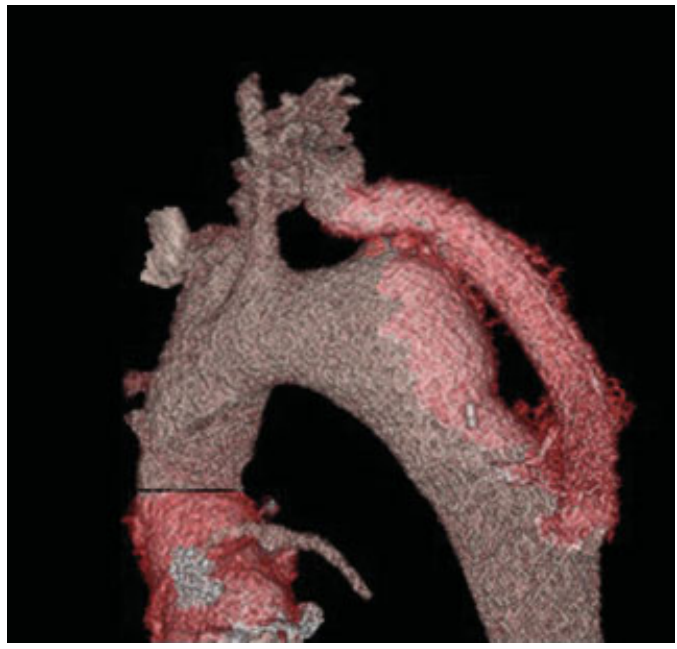

Fig. 5 Postoperative computed tomographic angiography of chest three-dimensional image, anterolateral view. Note resection of the aneurysm and graft to distal subclavian.

to symptomatic life-threatening vascular emergencies. Symptoms likely develop because of compression on adjacent structures and include brachial plexus irritation, Horner syndrome, dysphagia, hemoptysis, chest pain, and upper extremity deep vein thrombosis. ${ }^{5}$ These vascular anomalies cause significant morbidity including rupture, thrombosis, and peripheral embolization. ${ }^{6}$ Appropriate characterization of the aneurysm is achieved with noninvasive $\mathrm{CT}$ angiography as was done with our patient. Thorough workup should include evaluation for additional aneurysmal disease elsewhere in the body. The incidence of associated aneurysms ranges from 33 to $47 \%$, with a significant number of late deaths because of rupture of such aneurysms. ${ }^{7,8}$ Repair is undertaken to prevent these possible catastrophic sequela and to relieve symptoms. Surgical intervention is often challenging and complex due to by the presence of congenital anomalies, concomitant aortic pathology, prior interventions, comorbid conditions, or connective tissue diseases. ${ }^{8}$ Several approaches to surgical correction have been successful and have involved one or multiple operative stages. Each patient necessitates an individualized approach based on their unique anatomy, and that approach may include median sternotomy, axillary incision, supraclavicular incision, thoracotomy incision, or an endovascular and/or hybrid procedure. ${ }^{1}$

\section{Conclusion}

In conclusion, we successfully treated a symptomatic patient with a rare large left subclavian artery aneurysm, severely malfunctioning aortic valve, and aortic aneurysm through a two-stage open surgical correction.

\section{Note}

All authors have read the manuscript and approved the submission. The article has not been published anywhere and is not under consideration of publication in whole or in part.

\section{Conflict of Interest}

None.

\section{References}

1 Dougherty MJ, Calligaro KD, Savarese RP, DeLaurentis DA. Atherosclerotic aneurysm of the intrathoracic subclavian artery: a case report and review of the literature. J Vasc Surg 1995;21(3): 521-529

2 Dent TL, Lindenauer SM, Ernst CB, Fry WJ. Multiple arteriosclerotic arterial aneurysms. Arch Surg 1972;105(2): 338-344

3 Coselli JS, Crawford ES. Surgical treatment of aneurysms of the intrathoracic segment of the subclavian artery. Chest 1987;91(5): 704-708

4 Iida M, Hata H, Kimura H. A case of atherosclerotic aneurysm of the right subclavian artery with the right axillary arterial stenosis and enlargement of the ascending aorta. Ann Thorac Cardiovasc Surg 2011;17(6):599-602

5 Roh YN, Park KB, Do YS, Kim WS, Kim YW, Kim DI. A hybrid operation in a patient with complex right subclavian artery aneurysm. J Korean Surg Soc 2012;82(3):195-199

6 Niino T, Unosawa S, Shimura K. Intrathoracic left subclavian artery aneurysm: report of a case. Ann Vasc Dis 2012;5(1): $82-84$

7 Halldorsson A, Ramsey J, Gallagher C, Meyerrose G. Proximal left subclavian artery aneurysms: a case report and review of the literature. Angiology 2007;58(3):367-371

8 Marinho J, Pires A, Sousa G, Castela E. Right subclavian artery aneurysm in an adolescent with a bicuspid aortic valve. Pediatr Cardiol 2013;34(8):1952-1954 